\title{
Relativistic effect of gravitational deflection of light in binary pulsars
}

\author{
Oleg V. Doroshenko \\ Max-Plank-Institute fur Radioastronomie, Auf dem Hugel 71, D-53121 \\ Bonn, Germany and Astro Space Centre, RAS FIAN, Leninskii \\ Prospect, 53, Moscow 117924, Russia \\ Sergei M. Kopeikin \\ Laboratory of Astronomy and Geophysics, Hitotsubashi University, \\ Kunitachi, Tokyo 186, Japan and Astro Space Centre, RAS FIAN, \\ Leninskii Prospect, 53, Moscow 117924, Russia
}

Timing formula for data processing of observations of binary pulsars that accounts for the relativistic deflection of light in the gravitational field of the pulsar's companion is presented, and the measurability of this effect along with its variance estimates is discussed. The deflection of the pulsar's pulse trajectory in the gravitational field of its companion leads to variation in the pulsar's rotational phase. This variation appears as a narrow sharp growth of the magnitude of the post-fit residuals in the vicinity of the moment of the superior conjunction of the pulsar with its companion. In contrast to the relativistic Shapiro effect, the amplitude of the effect of gravitational deflection of the pulsar radio beam has two peaks with opposite signs, which become sharper as the inclination $i$ of the pulsar's orbit approaches to the right angle. The effect under consideration influences the estimation of parameters of the relativistic Shapiro effect in the binary pulsars with nearly edgewise orbits. Its inclusion in the fitting procedure provides a more careful measurement of the sine of the orbital inclination $i$, as well as the masses of the pulsar and its companion. This permits an improved testing of alternative theories of gravity in the strong field regime. The effect of the gravitational deflection of light has been numerically investigated for binary pulsars with nearly edgewise orbits. It is shown that the effect is observed in general only when cosi is less than 0.003 . This estimate becomes less restrictive as the pulsar's spin axis approaches the line of sight.

Further details can be obtained from the reference below.

\section{References}

O.V.Doroshenko \& S.M.Kopeikin, 1995, MNRAS, 274, 1029 